\title{
Tratamiento de la mordida cruzada anterior con plano inclinado anterior. Efecto sobre los arcos dentales
}

Carolina Rodríguez Manjarrés ${ }^{1}$, Jesús Alberto Hernández Silva

\section{Resumen}

Objetivo: Evaluar los cambios dimensionales de los arcos dentales primarios tratados con plano inclinado anterior como método de corrección de la mordida cruzada anterior. Métodos: Se trataron 10 pacientes con edades entre 3 y 5 años afectados con mordida cruzada anterior completa, se colocó un plano inclinado anterior elaborado en acrílico, que estuvo en posición en promedio 8.5 semanas. Se obtuvieron modelos de estudio en 3 momentos T0: antes del tratamiento; T1: 6 meses después de iniciado el tratamiento, y T2: al final del primer año de tratamiento y se evaluaron los cambios en las dimensiones de los arcos denta-

les. Resultados: En el 100\% de los pacientes se corrigió la mordida cruzada anterior, se encontraron diferencias estadísticamente significativas para las variables evaluadas con la terapéutica empleada. Conclusiones: El plano inclinado anterior produjo cambios dentales evidentes en un periodo corto de tiempo, sin recidivas y mejorando las dimensiones de los arcos dentales, especialmente el arco superior.

Palabras claves: Dentición primaria, mordida cruzada anterior, plano inclinado anterior, ortopedia funcional de los maxilares.

Relato de caso

\section{Tratamiento de la mordida cruzada anterior con plano inclinado anterior. Efecto sobre los arcos dentales}

\section{Resumo}

Objetivo: Avaliar as alterações dimensionais dos arcadas dentárias primárias tratados com plano inclinado como método de correção da mordida cruzada anterior. Métodos: 10 pacientes foram tratados com idades entre 3 e 5 sofrimento com mordida cruzada anterior completa, um plano inclinado em acrílico, que estava em posição de, em média, 8,5 semanas foi colocado. Modelos de estudo foram obtidos em 3 vezes T0: antes do tratamento; T1: 6 meses após o início do tratamento, e T2: o final do primeiro ano de tratamento e avaliadas alterações nas dimensões das arcadas dentárias. Resultados: Em 100\% dos pacientes a mordida cruzada anterior foi

\footnotetext{
${ }^{1}$ Residente Postgrado Odontología Pediátrica y Ortopedia Maxilar Escuela de Odontología Universidad del Valle (Cali, Colombia).

${ }^{2}$ Especialista en Odontología Integral del Niño y Ortopedia Maxilar de la Universidad de Antioquia. Profesor titular Escuela de Odontología Universidad del Valle (Cali, Colombia).
} 
corrigida, foram encontradas diferenças estatisticamente significativas para as variáveis avaliadas com o emprego terapêutico. Conclusões: O plano inclinado gerou alterações dentárias evidentes em pouco tempo, sem re- caídas e melhorando as dimensões dos arcos dentários, principalmente da arcada superior. Palavras-chave: Dentição primaria, mordida cruzada anterior, plano inclinado, ortopedia funcional dos maxilares.

\section{Treatment of anterior crossbite with lower inclined bite plane. Effect on the dental arches}

\section{Abstract}

Objective: To evaluate the dimensional changes of the primary dental arches treated with lower inclined bite plane as a method of correction of the anterior crossbite. Methods: Ten patients aged 3 to 5 years with complete anterior crossbite were treated with a lower inclined bite plane elaborated in acrylic. The average time of the plane position was 8.5 weeks. Dental casts were obtained in 3 moments T0: before treatment; T1: 6 months after starting treatment, and T2: at the end of the first year of treatment and changes in dimensions of dental arches were evaluated. Results: In $100 \%$ of patients the anterior crossbite were corrected, statistically significant differences for the variables evaluated with the therapeutic employed were found. Conclusions: The lower inclined bite plane generated evident dental changes in a short time, without relapses and improving the dimensions of the dental arches, especially the upper arch.

Keywords: Primary dentition, anterior crossbite, lower inclined bite plane, functional orthopedics of the jaw.

\section{Introducción}

La identificación de los diferentes factores de riesgo asociados al desarrollo de las maloclusiones es uno de los retos que enfrenta a diario el odontopediatra para brindar un diagnóstico temprano, y evitar que estas discrepancias se acentúen en la dentición mixta y permanente.

Según reporta la OMS, después de la caries dental y la enfermedad periodontal, las maloclusiones son consideradas como un problema de salud pública pues ocupan el tercer lugar de prevalencia. $^{1}$

Entre las maloclusiones más frecuentes que se pueden encontrar en la dentición primaria se reportan: apiñamiento dental, mordida abierta (anterior o posterior), mordida borde a borde, mordida profunda, mordida cruzada posterior y mordida cruzada anterior (MCA). ${ }^{2-4}$ Esta última puede definirse como el sobrepaso de los dientes anteriores inferiores por delante de los dientes anteriores superiores, relación que afecta el plano anteroposterior (foto 1), esta maloclusión puede tener un componente dentoalveolar, esquelético o funcional y puede afectar 1 o más dientes, ${ }^{5}$ siendo de canino a canino la de mayor 


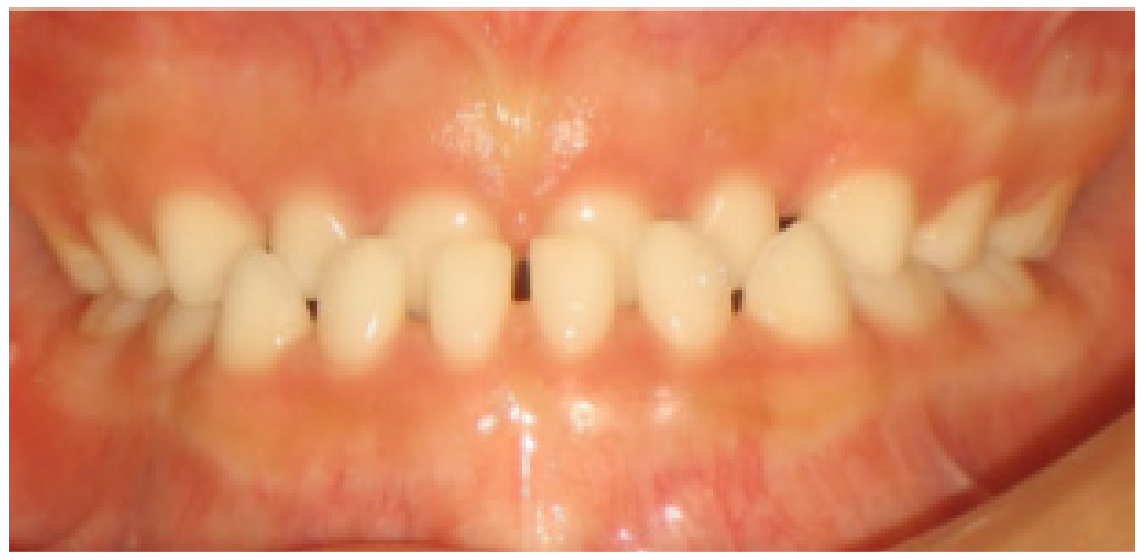

Foto 1. Mordida cruzada anterior en dentición primaria.

frecuencia. De acuerdo a diferentes estudios realizados se ha encontrado gran componente genético como agente etiológico principal de esta maloclusión. ${ }^{6-8}$

Estos pacientes presentan perfil recto o cóncavo, lengua baja y protrusiva y labio evertido, características que pueden variar según los componentes involucrados: Dentoalveolar puede verse afectado uno o más dientes, relación molar y canina Clase I, perfil facial recto y medidas cefalométricas normales; Esquelético presenta relación molar y canina Clase III, perfil cóncavo acompañado de retroquelia superior, mentón prominente y el tercio inferior esta disminuido. El análisis cefalométrico presenta variaciones en las medidas, registrando retrognatismo o micrognatismo del maxilar superior, prognatismo o macrognatismo mandibular o una combinación de alteraciones en tamaño y posición de ambos. La Pseudo clase III es de tipo funcional se caracteriza principalmente por la presencia de contactos prematuros que originan un desplazamiento mandibular en sentido anterior, se logra contacto borde a borde en relación céntrica, su perfil es recto o cóncavo; labio superior corto, disminución del tercio medio de la cara y la relación esquelética anteroposterior es normal. ${ }^{4,9-11}$
La prevalencia de la MCA es variable según reportan los diferentes estudios, ya que dependerá del tipo de estudio, la población a estudiar y el método de evaluación de la maloclusión. En poblaciones asiáticas oscila entre el 3-12\%, mientras que en americanos y europeos llega al 3\%. ${ }^{12,13}$ Según el Cuarto Estudio Nacional de Salud Bucal - ENSAB IV realizado en Colombia, en niños de 5 años se encontró una prevalencia del $2.41 \%{ }^{14}$

La mordida cruzada anterior debe ser tratada desde la dentición primaria para evitar repercusiones sobre el complejo craneofacial, crecimiento inadecuado de los maxilares y de sus componentes dentoalveolares, prevenir disfunciones a nivel de la articulación temporomandibular (ATM) por patrones musculares anormales o desviación funcional de la mandíbula; problemas periodontales a nivel de incisivos inferiores debido al trauma constante de la maloclusión, atriciones, movilidad, posibles patrones anormales de dicción y también para mejorar postura labial y la parte estética del paciente. ${ }^{15}$

La Ortopedia Funcional de los Maxilares favorece la corrección de las maloclusiones de forma temprana generando un cambio de postura terapéutico que ayuda a recuperar la excitación neuro-oclusal adecuada para lograr el equilibrio de todas las estructuras del sistema estomatognático. ${ }^{16}$ Los objetivos de tratamiento oportuno de la MCA serán lograr la corrección del overjet, restringir el crecimiento mandibular, estimular el desarrollo del maxilar superior, recuperar fisiología de la ATM y lograr paralelizar el plano oclusal ${ }^{17}$ y el plano de camper, que están con- 


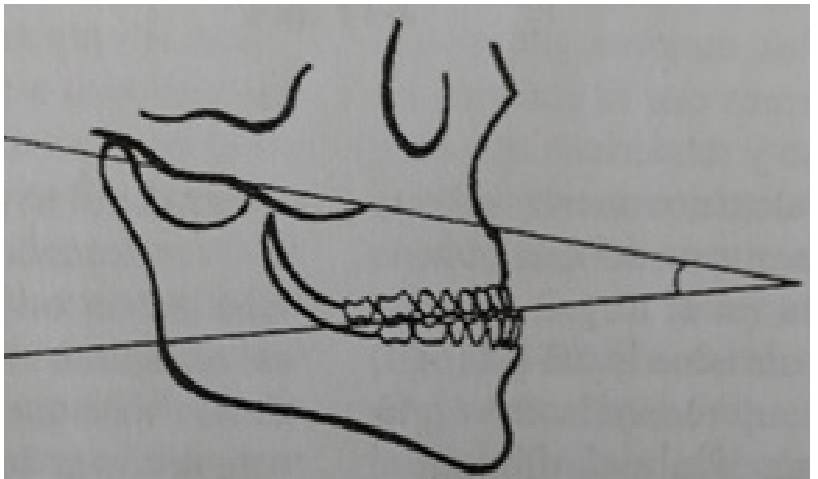

*Tomado de: Planas E. Rehabilitación Neuro-Oclusal (RNO). 2 edición. Editorial Amolca: 2008

Foto 2. Relación entre el plano de camper y el plano oclusal en la mordida cruzada anterior.

vergentes en esta maloclusión si se prolongan

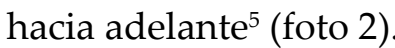

Dentro de las opciones de tratamiento de la MCA tenemos: Aparatología Ortopédica Funcional, Ortopedia Mecánica Extraoral, ${ }^{5}$ Desgaste Selectivo y/o Pistas Directas de Planas, ${ }^{18-20}$ Coronas Estéticas Pediátricas, ${ }^{21}$ Plano Inclinado Anterior, ${ }^{22-25}$ entre otras.

El plano inclinado anterior es utilizado para corregir un solo diente, o un segmento de dientes, usa como anclaje el arco dental inferior o superior, el movimiento es rápido y fisiológico, puesto que la fuerza ejercida es el resultado de una acción muscular normal. En el presente estudio utilizamos el plano inclinado anterior inferior confeccionado en acrílico, el cementado se realizó con Ionómero de vidrio. Debe tener una inclinación de $45^{\circ}$ para favorecer el cambio de postura y la inclinación axial de los dientes involucrados (foto 3). El plano inclinado anterior es bien aceptado por padres y pacientes, la adap- tación es rápida con recuperación pronta de las actividades funcionales del niño. ${ }^{5,7,22-26}$

El objetivo del presente estudio fue evaluar la efectividad del plano inclinado anterior en el tratamiento de la MCA en la dentición primaria y observar la estabilidad de los resultados alcanzados así como los efectos del tratamiento sobre las dimensiones de los arcos dentales medidas a los seis y a los doce meses de iniciado el estudio.

Este artículo es derivado de investigación, tesis de grado para optar al título de Especialista en Odontología Pediátrica y Ortopedia Maxilar, Escuela de Odontología Universidad del Valle.

\section{Materiales y metodos}

Se realizó un estudio de serie de casos con una muestra por conveniencia de 10 pacientes (4 niñas, 6 niños), rango de edad 3 a 5 años, en dentición primaria, con un periodo de lactancia materna en promedio de 6 meses, 4 presentaban antecedentes hereditarios de maloclusión clase III. A nivel dental se diagnosticó escalón mesial bilateral, relación canina clase III, mordida cruzada anterior completa de canino a canino, y al evaluar las interferencias caninas no se encon-

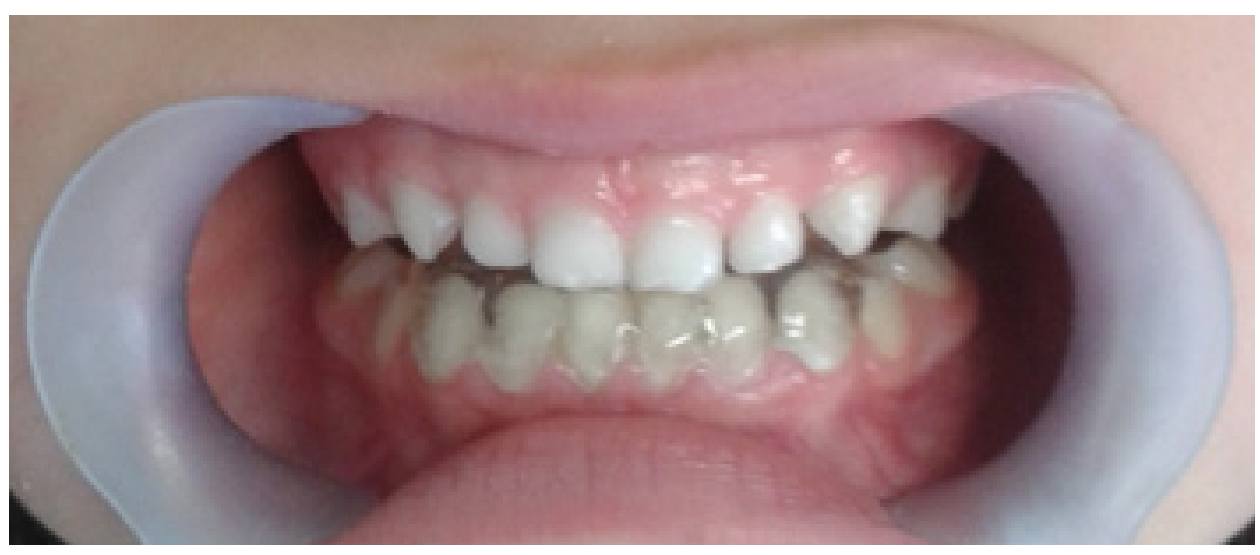

Foto 3. Plano anterior de mordida elaborado en acrílico. 
traron en la muestra seleccionada. Ninguno presentó otro tipo de maloclusión. Los pacientes no presentaban historia de caries ni presentaban hábitos orales y todos estaban sistémicamente sanos. Los pacientes que presentaban mordida cruzada que no cumplían los con los parámetros antes descritos no se incluyeron en este estudio.

A cada uno se le realizó toma de impresiones dentales en alginato (Orthoprint ${ }^{\circledR}$ Zhermack) para obtener modelos de estudio en 3 momentos: a) Modelo inicial antes del tratamiento; b) Modelo intermedio 6 meses después de iniciar tratamiento, y c) Modelo final al primer año de tratamiento. Sobre los modelos de estudio, se midieron las dimensiones de los arcos dentales con un calibrador digital (Vernier) y con dentímetro y alambre de cobre se evaluaron cambios dentoalveolares. Las medidas incluidas fueron: ancho intercanino, medida que corresponde a la distancia desde la cúspide de uno de los caninos a la cúspide del canino contralateral; ancho intermolar medido desde la zona media del tercio cervical lingual o palatino del segundo molar primario hasta su contralateral. Perímetro de arco: longitud de la línea curva que pasa sobre las cúspides vestibulares y los bordes incisales de los dientes, desde la superficie distal del segundo molar primario de un lado hasta la superficie distal del opuesto en el arco, esta medición se realizó con alambre de cobre marcada y posteriormente medida con una regla milimetrada. Longitud de arco, distancia entre dos tangentes una que toca el aspecto más vestibular de los incisivos en su punto medio, y la otra a la superficie distal de la corona de los segundos molares primarios. Overjet: se obtiene midiendo en sentido horizontal desde el borde incisal del inci- sivo central superior a borde incisal del incisivo central inferior, en pacientes con mordida cruzada anterior este valor es negativo y se expresa en milímetros. Y por último el overbite se obtuvo midiendo entre el borde incisal del diente incisivo central superior y el borde incisal del diente incisivo central inferior, expresado en porcentaje.

Las mediciones fueron tomadas por un (1) examinador, previamente estandarizado; para evidenciar el nivel de concordancia se empleó el coeficiente de correlación intraclase (CCI) y se logró un nivel alto de concordancia para cada una de las medidas de interés $(\mathrm{CCI}>0,8)$.

Los acudientes fueron informados sobre el objetivo del estudio, solicitando la participación del menor y aceptación con la firma del consentimiento informado. Este estudio cuenta con el aval del Comité Institucional de Revisión de Ética Humana y Experimentación Animal de la Facultad de Salud de la Universidad del Valle, Cali -Colombia. Igualmente se cumplió lo dispuesto en la Declaración de Helsinki y la Resolución No. 008430 de 1993 del Ministerio de Salud para investigación con seres humanos.

El tratamiento para la corrección de la mordida cruzada anterior en este estudio consistió en la elaboración y cementado de un plano inclinado

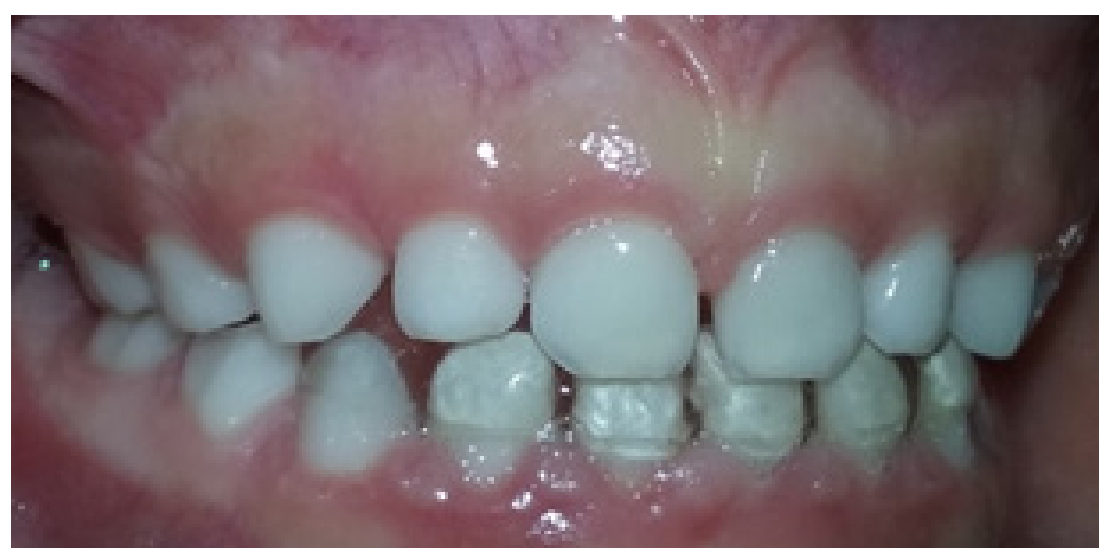

Foto 4. Plano anterior de mordida cementado (7 semanas aproximadamente en boca). 


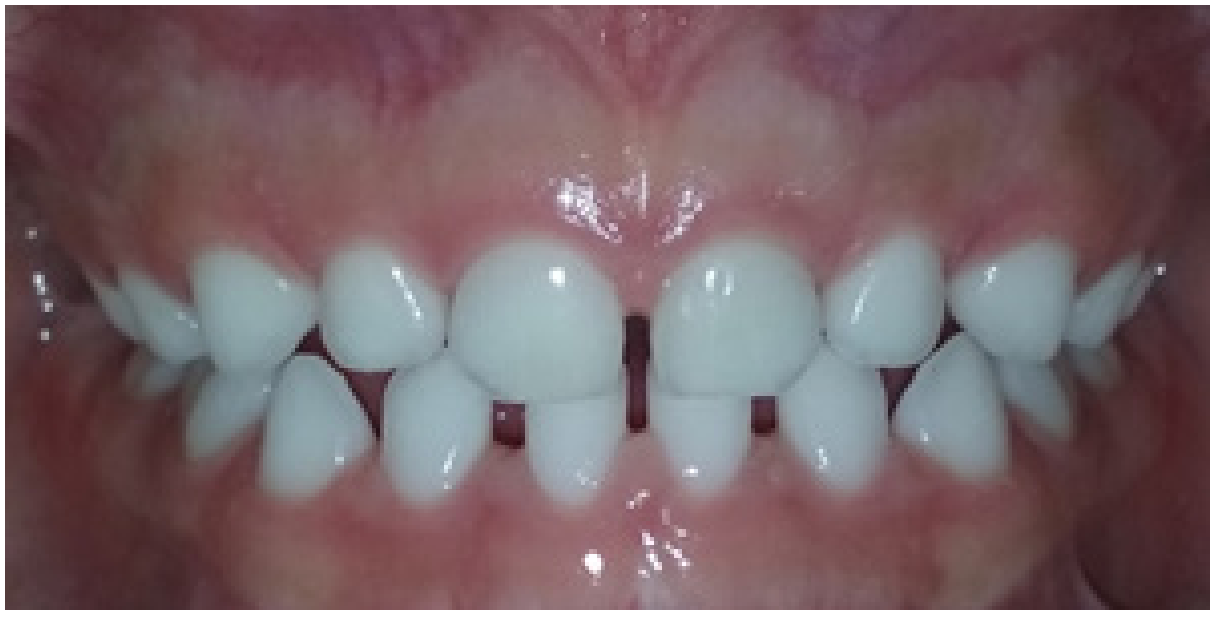

Foto 5. T2-Corrección de la maloclusión después del uso del plano anterior de mordida. tiempo promedio que el plano estuvo en posición vario entre 7 a 12 semanas promedio. Una vez logrado los objetivos, se retiró el plano inclinado anterior con una pinza para brackets y se eliminaron restos de material cementante (foto 5).

Análisis estadístico: Los registros de las medicioanterior en cada uno de los pacientes, elaborado en acrílico de autocurado, su extensión fue desde superficie distal de canino inferior derecho a superficie distal de canino inferior izquierdo, la inclinación del plano fue de 45 grados y la altura variaba en cada paciente según el grado de sobremordida (foto 4). Para cada plano se elaboraron los nichos que favorecieran la higiene oral, antes del cementado se realizó un aislamiento aplicando vaselina en todas las superficies dentales y con Ionómero de vidrio de autocurado marca Protech Ormco® se cementó, con papel de articular se verificó oclusión y presencia de posibles interferencias. Se dieron las recomendaciones a los acudientes de higiene oral y dieta. El primer control se realizó 8 días después y posteriormente se revisaron cada mes. El

\begin{tabular}{|c|c|c|c|}
\hline Variable & $\begin{array}{c}\text { Femenino } \\
(\mathbf{N}=4)\end{array}$ & $\begin{array}{c}\text { Masculi- } \\
\text { no }(\mathbf{N}=6)\end{array}$ & $\begin{array}{c}\text { General } \\
(\mathbf{N}=10)\end{array}$ \\
\hline $\begin{array}{c}\text { Edad T0 } \\
\text { (meses) }\end{array}$ & $42 \pm 6$ & $44 \pm 5$ & $43 \pm 5$ \\
\hline $\begin{array}{c}\text { Intervalo de } \\
\text { seguimiento } \\
\text { T0-T1 (meses) }\end{array}$ & $7 \pm 1$ & $7 \pm 1$ & $7 \pm 1$ \\
\hline $\begin{array}{c}\text { Intervalo de } \\
\text { seguimiento } \\
\text { T1-T2 (meses) }\end{array}$ & $6 \pm 1$ & $6 \pm 1$ & $6 \pm 1$ \\
\hline
\end{tabular}

*Test de Friedman (prueba exacta)

Tabla 1. Edad y tiempo de seguimiento de los pacientes bajo tratamiento de ortopedia maxilar. nes fueron consignadas directamente en una hoja de cálculo de Microsoft Excel y fueron importadas desde la aplicación SPSS versión 22 para el análisis estadístico, que consistió en el cálculo de las medidas de tendencia central y de dispersión en cada uno de los momentos de evaluación. Para determinar la existencia de diferencias estadísticamente significativas entre cada medición se utilizó la prueba de Friedman (exacta). Se estableció un nivel de confianza del $95 \%$ y de significancia del $5 \%$.

\section{Resultados}

Los diez niños participantes en el estudio corrigieron su MCA con el plano inclinado anterior

\begin{tabular}{|c|c|c|c|c|}
\hline Variable & $\mathrm{T} 0$ & $\mathrm{~T} 1$ & $\mathrm{~T} 2$ & $\mathrm{P}$ \\
\hline $\begin{array}{c}\text { Ancho } \\
\text { intercanino } \\
(\mathrm{mm})\end{array}$ & $29.39 \pm 2.21$ & $30.05 \pm 1.93$ & $30.49 \pm 1.89$ & $0.000^{*}$ \\
\hline $\begin{array}{c}\text { Ancho } \\
\text { intermolar } \\
(\mathrm{mm})\end{array}$ & $34.86 \pm 1.93$ & $35.36 \pm 1.89$ & $35.90 \pm 1.95$ & $0.000^{*}$ \\
\hline $\begin{array}{c}\text { Longitud } \\
\text { de arco } \\
(\mathrm{mm})\end{array}$ & $24.0 \pm 1.4$ & $24.5 \pm 1.4$ & $25.0 \pm 1.89$ & $0.020^{*}$ \\
\hline $\begin{array}{c}\text { Perímetro } \\
(\mathrm{mm})\end{array}$ & $76.7 \pm 2.4$ & $77.3 \pm 2.4$ & $78.1 \pm 2.2$ & $0.001^{*}$ \\
\hline
\end{tabular}

*Test de Friedman (prueba exacta)

Tabla 2. Distribución de las dimensiones del arco dental superior $(\mathrm{N}=10)$. 

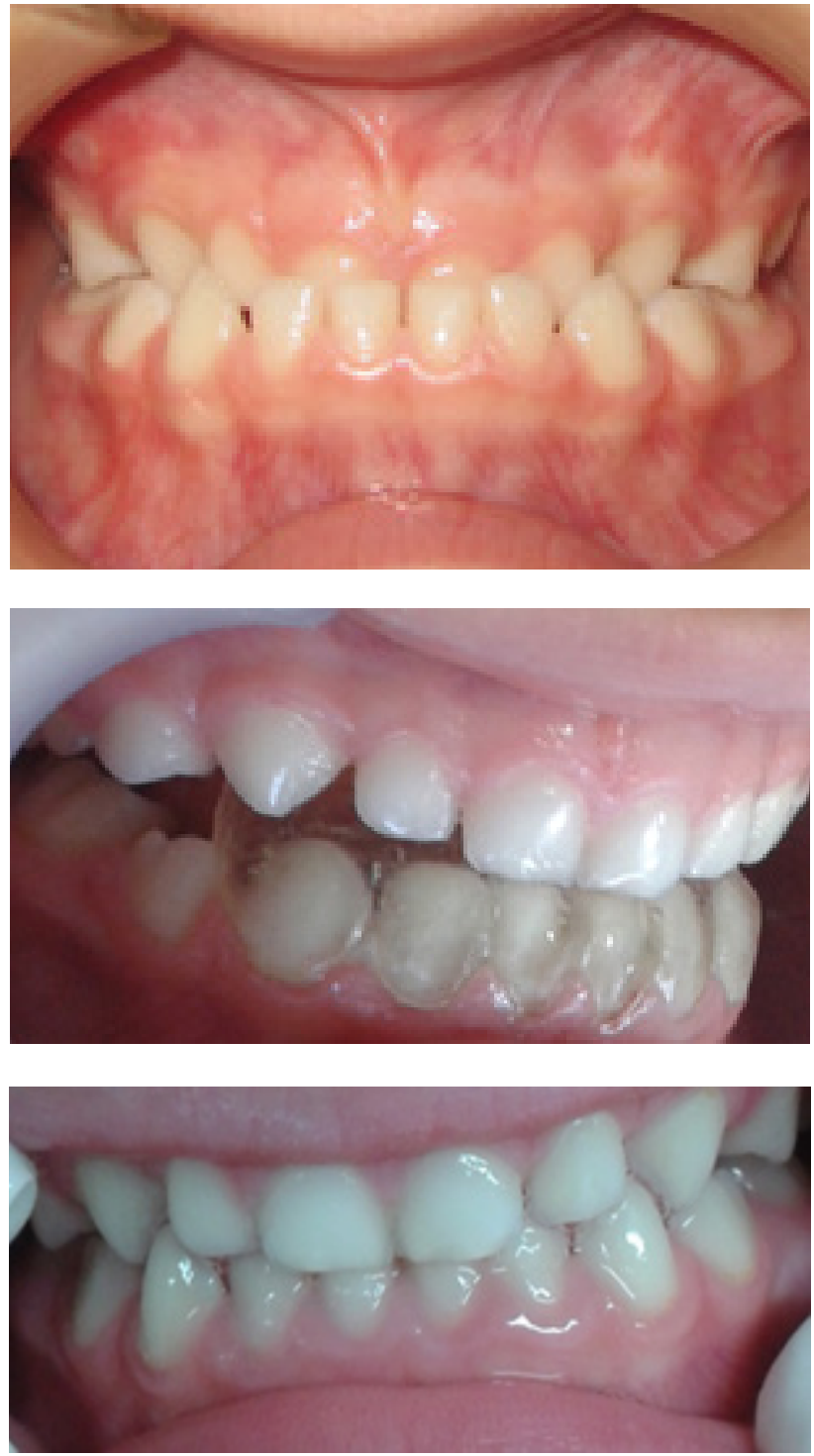

Foto 6. Secuencia paciente no. 7 correspondiente a T0 $-\mathrm{T} 1-\mathrm{T} 2$.

en un promedio de 8.5 semanas. La edad inicial de los pacientes fue de $43 \pm 5$ meses. La tabla 1 muestra los intervalos de seguimiento a los 6 meses (T0 - T1) y 1 año de tratamiento (T1 - T2).

En la tabla 2 se presentan los valores iniciales, intermedios y finales de los parámetros evaluados para el arco dental superior. El valor promedio inicial del ancho intercanino superior fue de 29,39 $\pm 2,21 \mathrm{~mm}$, intermedio 30,05 $\pm 1,93 \mathrm{~mm} \mathrm{y}$ final 30,49 $\pm 1,89 \mathrm{~mm}$. La distancia intermolar aumento $0,5 \mathrm{~mm}$ a los 6 meses y 1,04 $\mathrm{mm}$ al año de tratamiento. Se dio un aumento promedio de 1 $\mathrm{mm}$ en la longitud del arco entre T0 y T2. Los valores promedio del perímetro fueron inicial $76,7 \pm 2,4 \mathrm{~mm}$, intermedio $77,3 \pm 2,4 \mathrm{~mm}$ y final $78,1 \pm 2,2 \mathrm{~mm}$. Todas las diferencias fueron estadísticamente significativas.

En el arco dental inferior la media del ancho intercanino inicial fue de $23,94 \pm 1,10 \mathrm{~mm}$, final $24,40 \pm 0,90 \mathrm{~mm}$ y la intermolar inicial $29,18 \pm 1,25$ $\mathrm{mm}$, final 29,82 $\pm 1,34 \mathrm{~mm}$. El perímetro presento también una disminución de 1,6 $\mathrm{mm}$ al final del primer año de tratamiento (ver tabla 3). Todas las diferencias fueron estadísticamente significativas.

Los pacientes empezaron el tratamiento con un overjet promedio de $-1,1 \pm, 3 \mathrm{~mm}$ y overbite de $55,6 \% \pm 22,4 \%$. Durante el período de observación se encontraron cambios significativos en el overjet, el promedio general después de 1 año de tratamiento fue de $1,4 \pm 5 \mathrm{~mm}$. La tabla 4 presenta los cambios que ocurrieron en las variables anteriormente mencionadas. Todas las diferencias fueron estadísticamente significativas.

\section{Discusión}

La MCA se manifiesta desde edades muy tempranas y existe una amplia variedad de estudios que reportan el beneficio del tratamiento oportuno para este tipo de maloclusión. ${ }^{27}$ Pocos de estos trabajos han llevado un seguimiento longitudinal como el realizado en la presente investigación, donde se evidencian los cambios en las dimensiones de los arcos a los 6 y a los 12 meses de realizado el tratamiento. El llevar un seguimiento completo a un año permite reafirmar que los resultados encontrados generaron cambios tangibles en las diferentes variables evaluadas. 


\begin{tabular}{|c|c|c|c|c|}
\hline Variable & T0 & T1 & T2 & P \\
\hline $\begin{array}{c}\text { Ancho } \\
\text { intercanino } \\
(\mathrm{mm})\end{array}$ & $23.94 \pm 1.10$ & $24.39 \pm 1.02$ & $24.40 \pm .90$ & $0.007^{*}$ \\
\hline $\begin{array}{c}\text { Ancho } \\
\text { intermolar } \\
(\mathrm{mm})\end{array}$ & $29.18 \pm 1.25$ & $29.47 \pm 1.32$ & $29.82 \pm 1.34$ & $0.000^{*}$ \\
\hline $\begin{array}{c}\text { Longitud de } \\
\text { arco (mm) }\end{array}$ & $24.7 \pm 1.7$ & $24.3 \pm 1.7$ & $23.8 \pm 1.8$ & $0.040^{*}$ \\
\hline $\begin{array}{c}\text { Perímetro } \\
(\mathrm{mm})\end{array}$ & $73.1 \pm 2.3$ & $72.4 \pm 2.3$ & $71.5 \pm 2.1$ & $0.000^{*}$ \\
\hline
\end{tabular}

*Test de Friedman (prueba exacta)

Tabla 3. Distribución de las dimensiones del arco dental inferior $(N=10)$.

En los estudios acerca del desarrollo de la oclusión primaria realizados por Baume y otros autores, se plantea que en los arcos espaciados, cerrados y en los casos de maloclusión no hay cambios en las diferentes dimensiones (longitud, ancho intercanino e intermolar) entre los 3 y 5 1/2 años de edad, excepto si se someten a influencias ambientales. ${ }^{28-30}$ En nuestro estudio mediante la colocación de un plano inclinado anterior inferior se produjo un cambio de postura terapéutico, que favoreció el restablecimiento o recuperación de la excitación neural adecuada para permitirle al paciente realizar sus actividades fisiológicas con parámetros de normalidad. La recuperación funcional permitió evidenciar cambios en las dimensiones en los arcos dentales de los niños en los planos sagital, transversal y vertical de manera apreciable desde el punto de vista estadístico (ver tablas 2,3 y 4).

No se encontraron estudios de pacientes con mordida cruzada anterior en dentición primaria sin tratamiento. Los estudios de Williams y cols ${ }^{31}$ realizados en pacientes con normoclusión y rango de edad 3 a 5 años arrojo un perímetro superior de $74,47 \mathrm{~mm}$ en promedio y en el arco inferior $68,55 \mathrm{~mm}$ en promedio, en el presente estudio las mismas dimensiones tomadas un año después de realizado el tratamiento mostraron un promedio para el arco superior de $77,36 \mathrm{~mm}$ y para el arco inferior de $72,33 \mathrm{~mm}$.

\begin{tabular}{|c|c|c|c|c|}
\hline Variable & T0 & T1 & T2 & P \\
\hline $\begin{array}{c}\text { Overjet } \\
(\mathrm{mm})\end{array}$ & $-1.1 \pm .3$ & $1.0 \pm .3$ & $1.4 \pm .5$ & $0.000^{*}$ \\
\hline $\begin{array}{c}\text { Overbite } \\
(\%)\end{array}$ & $55.6 \pm 22.4$ & $36.1 \pm 9.8$ & $41.2 \pm 9.4$ & $0.000^{*}$ \\
\hline
\end{tabular}

*Test de Friedman (prueba exacta)

Tabla 4. Distribución de las dimensiones de Overjet y Overbite $(N=10)$.

El overjet cambió considerablemente siendo la medida promedio inicial de $-1,1 \pm 0,3 \mathrm{~mm}$, cambiando a los 6 meses $1,0 \pm 0,3 \mathrm{~mm}$ y al año 1,4 40,5 $\mathrm{mm}$, con una diferencia mayor para el género femenino, todos muy cerca de los parámetros aceptados como normales. ${ }^{32-33}$

El overbite promedio para T0 fue de 55,6\% 222,4 . Para T1 $36,1 \% \pm 9,8$ y la medida final promedio T2 fue $41,2 \% \pm 9,4$ ampliamente favorable para los pacientes tratados ortopédicamente.

\section{Conclusiones}

Los pacientes con mordida cruzada anterior generalmente presentan una estética pobre, una mordida bloqueada y pocos estímulos funcionales no favorables para el desarrollo armónico del complejo craneofacial.

El tratamiento temprano de la mordida cruzada anterior con el plano inclinado anterior fue determinante en la recuperación de la funcionalidad necesaria para corregir las desviaciones de crecimiento mencionadas y permitir un adecuado desarrollo de la dentición de los pacientes, los cambios dimensionales encontrados después del tratamiento muestran los beneficios en este sentido para la población estudiada.

El plano inclinado anterior como tratamiento temprano para la corrección de MCA en estos 
pacientes generó un cambio de postura terapéutico en la población evaluada.

En períodos variables pero relativamente cortos de 7 a 12 semanas se obtuvo una eficacia del tratamiento ortopédico del 100\% en la corrección de la MCA, y en el transcurso del año de duración del estudio se encontraron cambios di- mensionales estadísticamente significativos en ancho, longitud, perímetro, overjet y overbite.

\title{
Conflicto de interés
}

\author{
Los autores declaran no tener ningún conflicto \\ de interés.
}

\section{Referencias bibliográficas}

1. Pino IM, Véliz OL, García PA. Maloclusiones, según el índice de estética dental, en estudiantes de séptimo grado de Santa Clara. Medicentro Electrónica. 2014 Dic; 18(4): 177-179. [consultado 28 de mayo de 2016]. Disponible en: http:// scielo.sld.cu/scielo.php?script=sci_arttext\&pid=S1029-30432014000400007\&lng=es

2. Planells del Pozo P, De Nova MJ, Echaniz R, et al. Maloclusiones en dentición temporal. Odontología Pediátrica. 1993 Sept; 2(2): 77-82.

3. Iglesias IM. La salud oral en la dentición primaria: Estudio sobre la maloclusión y otras anomalías orales en una muestra de 441 niños de Vigo. Av. Odontoestomatol. 2003; 19(1): 11-19.

4. Alexander SA, Askari M, Lewis P. Occlusal Characteristics of the Primary Dentition Revisited. N Y State Dent J. 2015 Nov; 81(6): 34-39.

5. Hernández JA, Padilla MR. Tratamiento temprano de la mordida cruzada anterior. Revisión de la literatura. Rev. Estomat. 2011; 19(2): 40-47.

6. Toffol LD, Pavoni C, Baccetti T, Franchi L, Cozza P. Orthopedic treatment out- comes in Class III malocclusion. A systematic review. Angle Orthod. 2008; 78(3): 561-573.

7. Espinar E, Ruiz MB, Ortega H, et. al. Tratamiento Temprano de las Clases III. Rev Esp Ortod. 2011; 41: 79-89.

8. Negan P. Early Timely Treatment of Class III Malocclusion. Semin Orthod 2005; 11:140-145.

9. Da Silva L. Consideraciones Generales en el Diagnóstico y tratamiento de las Maloclusiones Clase III. Revista Latinoamericana de Ortodoncia y Odontopediatría. Ortodoncia.ws edición electrónica julio 2005. www.ortodoncia.ws. [consultado 31 de mayo de 2016]. Disponible en: https://www.ortodoncia.ws/publicaciones/2005/art14.asp

10. Ramírez J, Muñoz C, Gallegos A, Rueda MA. Maloclusión clase III. Salud en Tabasco 201016944-950. [consultado 2 de junio de 2016]. Disponible en: http://www.redalyc.org/articulo.oa?id=48720965007.

11. Velásquez V. Mordida cruzada anterior: diagnóstico y tratamiento con placa progenie. Revista Latinoamericana de Ortodoncia y Odontopediatría. 2005. www.ortodoncia.ws. [consultado 2 de junio de 2016]. Disponible en: https://www. ortodoncia.ws/publicaciones/2005/art8.asp

12. Solano B, Iglesias A, Yañez RM, Mendoza A, Alió JJ, Solano E. Maxillary protraction at early ages. The revolution of new bone anchorage appliances. J Clin Pediatr Dent. 2012 Winter;37(2): 219-229.

13. Xue F, Wong RW, Rabie AB. Genes, genetics, and Class III malocclusion. Orthod Craniofac Res. 2010 May;13(2):69-74. 14. Cuarto Estudio Nacional de Salud Bucal - ENSAB IV. [consultado 4 de junio de 2016]. Disponible en: https://www. minsalud.gov.co/sites/rid/Lists/BibliotecaDigital/RIDE/VS/PP/ENT/abc-salud-bucal.pdf

15. Park JH, Tai K, Ikeda M, Kanao A. Regaining leeway space and anterior crossbite correction with a modified maxillary molar distalizing appliance. J Clin Pediatr Dent. 2013 Spring; 37(3):329-234.

16. Simoes WA. Ortopedia Funcional de los Maxilares. A través de la Rehabilitación Neuro - Oclusal. Tercera edición. Artes Médicas Latinoamérica: São Paulo; 2004.

17. Raymond JL, Pimentel I, Vignolo R. Tratamiento ortopédico de las maloclusiones de clase III: masticación y plano oclusal. Ortodoncia Clínica 2009;12(4):186-193. 
18. Ramírez GO. Planas Direct Tracks for Early Crossbite Correction. J Clin Orthod. 2003 Jun; 37(6):294-8.

19. Gribel MN. Planas Direct Tracks in the early treatment of unilateral crossbite with mandibular postural deviation. Why worry so soon? World J Orthod. 2002; 3(3):239-249.

20. Dos Santos RR, Isper AJ, Saliba CA. Early Correction of Malocclusion Using Planas Direct Tracks. Case Reports in Dentistry. vol. 2013, Article ID 395784, 2013. doi:10.1155/2013/395784

21. Ramirez G. Treatment of anterior crossbite in the primary dentition with esthetic crowns: report of 3 cases. Pediatr Dent. 2011;33(4):339-42.

22. Hernández JA, Villavicencio JE. Un método de tratamiento para la mordida cruzada anterior en la dentición primaria. Rev. Estomat. 1997; 7(1): 48-51.

23. Croll TP. Fixed inclined plane correction of anterior cross bite of the primary dentition. J Pedod. 1984; 9 (1): $84-94$.

24. Croll TP, Riesenberger RE. Anterior crossbite correction in the primary dentition using fixed inclined planes. I. Technique and examples. Quintessence Int. 1987; 18(12):847-53.

25. Quirós O. El plano inclinado para el tratamiento de la mordida cruzada anterior. Acta Odontológica Venezolana 2003; 40 (3). [consultado 5 de junio de 2016]. Disponible en: http://www.actaodontologica.com/ediciones/2002/3/plano_ inclinado_mordida_cruzada_anterior.asp

26. Josefa P, Medina P. Adaptación del niño a la consulta odontológica. Acta Odontológica Venezolana 1998;36(2). [consultado 6 de junio de 2016]. Disponible en: http://www.actaodontologica.com/ediciones/1998/2/adaptacion_nino_ consulta_odontologica.asp

27. Alzate J, Álvarez E, Botero P. Tratamiento Temprano de la Maloclusión Clase III con aparatología Ortopédica: Reporte de caso con 7 años de control. Rev ALOP. 2014; 4(1): 95-109. [consultado 7 de junio de 2016]. Disponible en: http://www.revistaodontopediatria.org

28. Baume LJ. Phisiological tooth migration and its significance for the development of occlusion. Part I. The biogenetic course of deciduous dentition. J Dent. Res. 1950 Apr;29(2):123-32.

29. Lewis SJ, Lehman IA. Observations on growth changes of the teeth and dental arches. Dent Cosmos. 1929; 71:480-99. 30. Goldstein MS, Stanton FL. Changes in Dimensions and Form of the Dental Arches With Age. Int. J. Orthodontia. 1935; 21:357-380.

31. Williams FD, Valverde R, Meneses A. Dimensiones de arcos y relaciones oclusales en dentición decidua completa. Rev Estomatol Herediana 2004;14(1-2): 22-16.

32. Foster TD, Hamilton MC. Occlusion in the primary dentition. Br Dent J. 1969; 21: 77-9.

33. Serna CM, Silva R. Características de la oclusión en niños con dentición primaria de la Ciudad de México. Revista ADM 2005; 62(2):45-51.

Recibido: 19/09/2016

Acrptado: 18/11/2016

Autor correspondiente: Carolina Rodríguez Manjarrés carordgz86@hotmail.com

Dirección: calle 5 \# 66B-69 apto 902. Cali-Colombia. Teléfono: 3104286800 